\title{
Metrics induced by capacities and boundary behaviors of quasiconformal mappings on open Riemann surfaces
}

By Hiroshi TANAKA*

(Received June 16, 1975)

\section{Introduction.}

M. Nakai (cf. [4]) proved that every quasiconformal mapping between two open Riemann surfaces can be homeomorphically extended to their Royden compactifications. It is well-known (cf. [2]) that the Royden compactification is not metrizable. In this paper we shall study the homeomorphic extensibility of quasiconformal mappings between two open Riemann surfaces to their metrizable compactifications. To do so we shall introduce a new metric $d=d_{R}$ on an open Riemann surface $R$ induced by the Kuramochi capacity on $R$. Our main results are the followings:

(i) Let $R$ be an open Riemann surface. If each Kuramochi kernel $\tilde{g}_{b}$ with pole $b$ on the Kuramochi boundary of $R$ is unbounded, then the completion of $R$ with respect to $d$ is compact.

(ii) Let $R_{1}$ and $R_{2}$ be two open Riemann surfaces. If both $R_{1}$ and $R_{2}$ satisfy the assumption in (i), then every quasiconformal mapping from $R_{1}$ onto $R_{2}$ can be homeomorphically extended over their completions with respect to $d$.

\section{Metrics induced by capacities.}

Let $R$ be an open Riemann surface. We say that a closed curve in $R$ joining $a \in R$ and $b \in R$ means a continuous mapping $\gamma: z=z(t)$ of $[0,1]$ into $R$ such that $z(0)=a$ and $z(1)=b$. We write $r=\{z(t) ; 0 \leqq t \leqq 1\}$ for simplicity. We denote by $\Gamma_{a, b}=\Gamma_{a, b}(R)$ the family of all closed curves in $R$ joining $a$ and $b$.

A non-negative finite real-valued function $\Phi$ on the family of all compact subsets on $R$ is said to be a capacity in the sense of $G$. Choquet if it satisfies the following properties:

(a) If $K_{1} \subset K_{2}$, then $\Phi\left(K_{1}\right) \leqq \Phi\left(K_{2}\right)$.

(b) $\Phi\left(K_{1} \cup K_{2}\right)+\Phi\left(K_{1} \cap K_{2}\right) \leqq \Phi\left(K_{1}\right)+\Phi\left(K_{2}\right)$.

*) 田 中 博 
(c) Given a compact subset $K$ of $R$ and any $\varepsilon>0$, there is an open set $G$ in $R$ such that any compact subset $K^{\prime}$ with $K \subset K^{\prime} \subset G$ implies $\Phi\left(K^{\prime}\right)<\Phi(K)+\varepsilon$.

Definition 1. For $a, b \in R$, we set

$$
\mu(a, b)=\inf \left\{\Phi(\gamma) ; \gamma \in \Gamma_{a, b}(R)\right\} .
$$

Proposition 1.

(i) $\mu$ is a pseudometric on $R$, i.e.,

(1) $0 \leqq \mu(a, b)<\infty, a=b \Rightarrow \mu(a, b)=0$.

(2) $\mu(a, b)=\mu(b, a)$.

(3) $\mu(a, b) \leqq \mu(a, c)+\mu(c, b)$.

(ii) $\mu(a, b)=\inf \{\Phi(K)$; where $K$ runs over all continua in $R$ containing both $a$ and $b$.

PROof. The proof of (i) follows immediately from the properties $(a),(b)$ and $(c)$ of capacity.

(ii) Let $a$ and $b$ any points in $R$. We denote by $\nu(a, b)$ the right hand side in (i). Since any closed curve is a continuum, we obtain that $\nu(a, b) \leqq \mu(a, b)$. For any $\varepsilon>0$, there is a continuum $E$ in $R$ containing both $a$ and $b$ such that $\Phi(E)<\nu(a, b)+\varepsilon$. Since $E$ is a connected compact set, we can find a domain $G$ (=a connected open set) on $R$ such that $E \subset G$ and any compact set $F$ with $E \subset F \subset G$ implies $\Phi(E) \leqq \Phi(F)<\Phi(E)+\varepsilon$. Since $G$ is connected and contains both $a$ and $b$, there is a closed curve $\gamma$ in $G$ joining both $a$ and $b$. If we set $F=E \cup r$, then we obtain that

$$
\mu(a, b) \leqq \Phi(\gamma) \leqq \Phi(E \cup \gamma)<\Phi(E)+\varepsilon<\nu(a, b)+2 \varepsilon .
$$

Since $\varepsilon$ is arbitrary, we complete the proof.

Some special metrics induced by capacities

Definition 2 ([1]). Let $R$ be a hyperbolic Riemann surface. Let $\boldsymbol{z}$ be a fixed point in $R$. For a compact subset $K$ of $R-\{z\}$, we denote by $\omega_{z}(K)$ the harmonic measure of $\partial K$ with respect to $R-K$ at $z$. For $a, b \in$ $R-\{z\}$, we set

$$
\mu(a, b)=H_{z}(a, b)=\inf \left\{\omega_{z}(\gamma) ; \gamma \in \Gamma_{a, b}(R-\{z\}) .\right.
$$

It is known [1] that this $\mu(a, b)$ is a metric on $R-\{z\}$. Furthermore the topology induced by $\mu$ is compatible with the original topology on $R-\{z\}$.

Definition 3. Let $R$ be a hyperbolic Riemann surface and let $C=C_{R}$ be the Green capacity on $R$. For $a, b \in R$, let

$$
\mu(a, b)=\rho(a, b)=\rho_{R}(a, b)=\inf \left\{C(\gamma) ; \gamma \in \Gamma_{a, b}(R)\right\} .
$$


Definition 4. Let $R$ be an open Riemann surface. Let $K_{0}$ be a closed disk in $R$ and let $R_{0}=R-K_{0}$. Let $\widetilde{C}$ be the Kuramochi capacity on $R_{0}$ (cf. [2]). For $a, b \in R_{0}$, let

$$
\mu(a, b)=d(a, b)=d_{R_{0}}(a, b)=\inf \left\{\widetilde{C}(\gamma) ; \gamma \in \Gamma_{a, b}\left(R_{0}\right)\right\} .
$$

By elementary properties of Green capacity and Kuramochi capacity, we obtain the following lemma.

Lemma 1. (i) Let $R$ be a hyperbolic Riemann surface and $G$ be a domain on $R$. Then

$$
\rho_{R}(a, b) \leqq \rho_{\theta}(a, b) \text { for } a, b \in G .
$$

(ii) Let $R$ be an open Riemann surface. Let $K_{0}$ be a closed disk in $R$ and let $R_{0}=R-K_{0}$. Then

$$
d_{R_{0}}(a, b) \leqq \rho_{R_{0}}(a, b) \text { for } a, b \in R_{0} .
$$

(iii) Let $R$ be an open Riemann surface. Let $K_{0}, K_{0}^{\prime}$ be closed disks in $R$ with $K_{0} \subset K_{0}^{\prime}$. Set $R_{0}=R-K_{0}$ and $R_{0}^{\prime}=R-K_{0}^{\prime}$. Then

$$
d_{R_{0}}(a, b) \leqq d_{R_{0}}(a, b) \text { for } a, b \in R_{0}^{\prime} .
$$

\section{The metric $\rho$.} $a, b \in U$.

LEMMA 2. Let $U=\{|z|<1\}$. Then $\rho_{D}(a, b)=C_{D}\left(\left[0,\left|\frac{b-a}{1-a b}\right|\right]\right)$ for

Proof. We may assume that $a \neq b$.

(i) Suppose $a=0$. Let $\gamma$ be any curve in $\Gamma_{0, b}(U)$. Then it follows from Hilfssatz 19.1 in $[3]$ that $C_{\sigma}([0,|b|]) \leqq C_{\sigma}(\gamma)$. Hence we see that $\rho_{\sigma}(0$, $b)=C_{b}([0,|b|])$.

(ii) Suppose $a$ is arbitrary. Then we can find a linear transformation $w=T(z)$ of $U$ onto itself such that $T(0)=0$ and $T(b)=\left|\frac{b-a}{1-\bar{a} b}\right|$. Since $\rho_{V}$ is invariant under conformal mappings, it follows from (i) that

$$
\rho_{V}(a, b)=\rho_{V}(T(a), T(b))=\rho_{U}\left(0,\left|\frac{b-a}{1-a b}\right|\right)=C_{V}\left(\left[0,\left|\frac{b-a}{1-a b}\right|\right]\right) .
$$

Corollary (Schwartz lemma). Every analytic mapping $f: U \rightarrow U$ is distancedecreasing, i.e., satisfies

$$
\boldsymbol{\rho}_{\boldsymbol{V}}(f(a), f(b)) \leqq \boldsymbol{\rho}_{\sigma}(a, b) \quad(a, b \in U)
$$

and the equality is valid if and only if $f$ is an automorphism of $U$.

PRoposition 2. (i) $\rho_{V}$ is a metric on $U$.

(ii) The metric $\rho_{D}$ is compatible with the topology on $U$. 
Proof. By properties of capacity and Lemma 2, we have (i). Furthermore it follows from Lemma 2 that (ii) is valid.

For $a, b \in U$, we set

$$
\sigma(a, b)=\frac{1}{2} \log \frac{1+r}{1-r}
$$

where $r=\left|\frac{b-a}{1-a b}\right|$. The function $\sigma(a, b)$ is called a non-euclidean metric on $U$.

Proposition 3. For a fixed $a \in U$, we have

$$
\lim _{b \rightarrow \xi} \frac{\rho_{V}(a, b)}{\sigma(a, b)}=\frac{4}{\pi^{2}}
$$

uniformly in $\xi \in \partial U$.

Proof. For a fixed $a \in U$ and an arbitrary $b \in U$, let $r=\left|\frac{b-a}{1-a b}\right|$. If $b$ tends to a boundary point $\xi \in \partial U$, then $r$ tends to 1 . Thus it follows from [3] that

$$
\rho_{\delta}(a, b)=\frac{2}{\pi^{2}} \log \frac{8}{1-r}+O(1) \text { as } \quad r \rightarrow 1 .
$$

Hence we have

$$
\rho_{U}(a, b)=\frac{2}{\pi^{2}} \log \frac{1}{1-r}+O(1) \text { as } \quad r \rightarrow 1 .
$$

Since $\sigma(a, b)=\frac{1}{2} \log \frac{1}{1-r}+O(1)$, we complete the proof.

Corollary. For $t(0<t<1)$, we set $D_{t}=\{|a|<t\}$. Then, for any $t_{1}\left(0<t_{1}<1\right)$ and $c>\frac{4}{\pi^{2}}$, there exists $t_{2}\left(t_{1}<t_{2}<1\right)$ such that

$$
c^{-1} \sigma(a, b) \leqq \rho_{\sigma}(a, b) \leqq c \sigma(a, b)
$$

for any $a \in D_{t_{1}}$ and $b \in D-\bar{D}_{t_{2}}$.

LEMMA 3. Let $R$ be an open Riemann surface and $K_{0}$ be a closed disk on $R$. Let $U$ be an open disk on $R$ with $U \cap K_{0}=\emptyset$. Let $K$ be a compact subset of $U$ such that $U-K$ is connected. Let $\left\{K_{n}\right\}_{n=1}^{\infty}$ be a family of compact subsets of $K$. Then the following properties are equivalent each other.

(a) $C_{V}\left(K_{n}\right) \rightarrow 0$ as $n \rightarrow \infty$.

(b) $C_{\theta}\left(K_{n}\right) \rightarrow 0$ as $n \rightarrow \infty$ for any hyperbolic domain $G$ on $R$ with $U \subset G$.

(c) $\widetilde{C}\left(K_{n}\right) \rightarrow 0$ as $n \rightarrow \infty$. 
PROOF. Let $u_{n}$ (resp. $\left.v_{n}\right)$ be the harmonic measure of $\partial K_{n}$ with respect to $U-K_{n}$ (resp. $G-K_{n}$ ). Then it is easy to see that $C_{U}\left(K_{n}\right)\left(\right.$ resp. $C_{\theta}\left(K_{n}\right)$, $\left.\widetilde{C}\left(K_{n}\right)\right) \rightarrow 0$ as $n \rightarrow \infty$ if and only if $u_{n}$ (resp. $\left.v_{n}, 1_{\widetilde{K}_{n}}\right)^{1)}$ converges to zero locally uniformly in $U-K$ (resp. $G-K, R_{0}-K$ ) as $n \rightarrow \infty$. Since $C_{\theta}\left(K_{n}\right) \leqq$ $C_{U}\left(K_{n}\right), \widetilde{C}\left(K_{n}\right) \leqq C_{R_{0}}\left(K_{n}\right)$ and $v_{n} \leqq 1_{\widetilde{\aleph}_{n}}$ in $U-K(n=1,2, \cdots)$, we complete the proof.

TheOREm 1. Suppose $R$ is a hyperbolic Riemann surface. Then

(i) $\rho_{R}$ is a metric on $R$.

(ii) The metric $\rho_{R}$ is compatible with the topology on $R$.

Proof. We set $\boldsymbol{\rho}=\boldsymbol{\rho}_{R}$ in the following. Since $\boldsymbol{\rho}$ is a pseudometric, it is sufficient to prove that $\rho(a, b)=0$ implies $a=b$. If this were not the case, then we could find $a$ and $b$ in $R$ such that $\rho(a, b)=0$ and $a \neq b$. Then we can find a parametric disk $U$ on $R$ with center at $a$ such that $b \in U$. Since $a \neq b, \rho_{v}(a, b)>0$. On the other hand, since $\rho(a, b)=0$, there is a subfamily $\left\{\gamma_{n}\right\}_{n=1}^{\infty}$ of $\Gamma_{a, b}$ such that $C_{R}\left(\gamma_{n}\right) \rightarrow 0$ as $n \rightarrow \infty$. Let $\zeta=\phi(z)$ be a local parameter on $U$ such that $\phi(a)=0$ and $\phi(U)=\{|\zeta|<1\}$. For each $n$, we can find a subcurve $\gamma_{n}^{\prime}$ of $\gamma_{n}$ on $\phi^{-1}(|\zeta| \leqq|\psi(b)|)$ which connects $a$ and a point of $\psi^{-1}(|\psi(b)|)$. Then $0<\rho_{V}(a, b) \leqq C_{V}\left(\gamma_{n}^{\prime}\right)$. Since $C_{R}\left(\gamma_{n}^{\prime}\right) \leqq C_{R}\left(\gamma_{n}\right)$ $\rightarrow 0$ as $n \rightarrow \infty$, it follows from Lemma 3 that $C_{b}\left(\gamma_{n}^{\prime}\right) \rightarrow 0$ as $n \rightarrow \infty$. This is a contradiction. Thus $\rho(a, b)=0$ implies $a=b$.

(ii) Let $\left\{a_{n}\right\}_{n=1}^{\infty}$ be a sequence in $R$ which tends to $a \in R$ as $n \rightarrow \infty$. Let $U$ be a parametric disk in $R$ with center at $a$. Then there is an $n_{0}$ such that $a_{n} \in U$ if $n \geqq n_{0}$. Since $0 \leqq \rho_{R}\left(a, a_{n}\right) \leqq \rho_{U}\left(a, a_{n}\right) \rightarrow 0$ as $n \rightarrow \infty$ by Proposition 1, we see that $\rho_{R}\left(a, a_{n}\right) \rightarrow 0$ as $n \rightarrow \infty$. Conversely suppose $a$ is a point in $R$ and $\left\{a_{n}\right\}_{n=1}^{\infty}$ is a sequence in $R$ such that $\rho\left(a, a_{n}\right) \rightarrow 0$ as $n \rightarrow \infty$. Then there are a parametric disk $U$ with center at $a$ which corresponds to a unit disk $\{|\zeta|<1\}$ and a subsequence $\left\{a_{n_{k}}\right\}_{k=1}^{\infty}$ of $\left\{a_{n}\right\}_{n=1}^{\infty}$ such that each $a_{n_{k}}$ does not belong to $U$. Let $\zeta=\phi(z)$ be a local parameter on $U$ such that $\phi(a)=0$ and $\phi(U)=\{|\zeta|<1\}$. Since $\rho\left(a, a_{n}\right) \rightarrow 0$ as $n \rightarrow \infty$, we can find $\gamma_{n} \in \Gamma_{a, a_{n}}(n=1,2, \cdots)$ such that $C_{R}\left(\gamma_{n}\right) \rightarrow 0$ as $n \rightarrow \infty$. For each $n_{k}$, we can find a subcurve $\gamma_{n_{k}}^{\prime}$ of $\gamma_{n_{k}}$ which connects $a$ and a point $b_{n_{k}}$ of $\psi^{-1}(|\zeta|=$ $1 / 2)$ on $\psi^{-1}(|\zeta| \leqq 1 / 2)$. Since $C_{R}\left(\gamma_{n_{k}}^{\prime}\right) \leqq C_{R}\left(\gamma_{n_{k}}\right) \rightarrow 0$ as $k \rightarrow \infty$, it follows from Lemma 3 that $C_{V}\left(\gamma_{n_{k}}^{\prime}\right) \rightarrow 0$ as $k \rightarrow \infty$. On the other hand, we obtain that $0<\boldsymbol{\rho}_{\psi(U)}(0,1 / 2)=\boldsymbol{\rho}_{U}\left(a, b_{n_{k}}\right) \leqq C_{U}\left(\gamma_{n_{k}}^{\prime}\right)$. This is a contradiction. Therefore $a_{n}$ $\rightarrow a$ as $n \rightarrow \infty$.

1) See p. 163 in [2] for the definition. 


\section{The metric $d$ and quasiconformal mappings.}

Let $R$ be an open Riemann surface. Let $K_{0}$ be a closed disk in $R$ and let $R_{0}=R-K_{0}$. Let $\widetilde{C}$ be the Kuramochi capacity on $R_{0}$ (cf. [2]).

THEOREM 2. (i) $d$ is a metric on $R_{0}$.

(ii) The metric $d$ is compatible with the topology on $R_{0}$.

Proof. (i) Suppose $d(a, b)=0$ for some two distinct points $a$ and $b$ of $R_{0}$. Then there exists a parametric disk $U$ with center at $a$ such that $b \in U$. Let $\zeta=\phi(z)$ be a local parameter on $U$ such that $\phi(a)=0$ and $\phi(U)$ $=\{|\zeta|<1\}$. Since $d(a, b)=0$, there is a sequence $\left\{\gamma_{n}\right\}_{n=1}^{\infty}$ in $\Gamma_{a, b}\left(R_{0}\right)$ such that $\widetilde{C}\left(\gamma_{n}\right) \rightarrow 0$ as $n \rightarrow \infty$. For each $n$, we can find a subcurve $\gamma_{n}^{\prime}$ of $\gamma_{n}$ which connects $a$ and a point $b_{n}$ of $\psi^{-1}(|\zeta| \leqq|\psi(b)|)$ on $\psi^{-1}(|\zeta| \leqq|\psi(b)|)$. Since $\widetilde{C}\left(\gamma_{n}^{\prime}\right) \rightarrow 0$ as $n \rightarrow \infty$, it follows from Lemma 3 that $0<\boldsymbol{\rho}_{\psi(U)}(0, \phi(b))=\boldsymbol{\rho}_{\sigma}\left(a, b_{n}\right)$ $\leqq C_{U}\left(\gamma_{n}^{\prime}\right) \rightarrow 0$ as $n \rightarrow \infty$. This is a contradiction. Thus $d$ is a metric.

The proof of (ii) can be proved by an analogous argument to the one of (ii) in Theorem 1 .

We refer to [4] for the definition and properties of quasiconformal mapping. Let $R_{1}, R_{2}$ be open Riemann surfaces and $\phi$ be a quasiconformal mapping of $R_{1}$ onto $R_{2}$. We denote by $B C D\left(R_{i}\right)$ the family of all bounded continuous Dirichlet functions on $R_{i}(i=1,2)$ (cf. [2]). For $f \in B C D\left(R_{i}\right)$, we denote by $\|f\|_{R_{i}}^{2}$ the Dirichlet integral of $f$ on $R_{i}(i=1,2)$. As for quasiconformal mappings and Dirichlet functions, the following theorem is wellknown.

THEOREM 3 (cf. [4]). Let $\phi$ be a quasiconformal mapping of $R_{1}$ onto $R_{2}$. Then

$$
1 / K(\phi)\|f\|_{R_{1}}^{2} \leqq\left\|f^{\circ} \phi^{-1}\right\|_{R_{2}}^{2} \leqq K(\phi)\|f\|_{R_{1}}^{2}
$$

for each $f \in B C D\left(R_{1}\right)$, where $K(\phi)$ is the maximum dilatation of $\phi$.

Let $K_{0}$ be a closed disk in $R_{1}$ and $K_{0}^{\prime}=\phi\left(K_{0}\right)$. Then the following theorem follows from Theorem 3 and Satz 17.6 in [2].

THEOREM $4 . \quad 1 / K(\phi) \widetilde{C}(E) \leqq \widetilde{C}^{\prime}(\phi(E)) \leqq K(\phi) \widetilde{C}(E)$

for any compact subset $E$ of $R_{1}-K_{0}$, where $\widetilde{C}^{\prime}$ is the Kuramochi capacity on $R_{2}-K_{0}^{\prime}$.

Proof. Let $E$ be an arbitrary compact subset of $R_{1}-K_{0}$. Let $F$ be any regular compact subset ${ }^{2}$ of $R_{1}-K_{0}$ such that $E \subset F$. We set

2) We say that a compact set is regular if its relative boundary consists of a finite number of analytic arcs. 


$$
f=\widetilde{p}^{x^{F^{\prime}}} \text { on } R_{1}-K_{0} \text { and }=0 \text { on } K_{0 .}{ }^{3)}
$$

Then $f \in B C D\left(R_{1}\right)$ and $f \circ \phi^{-1} \in B C D\left(R_{2}\right)$. Since $f \circ \phi^{-1}=1$ on $\phi(F)(\supset \phi(E))$ and $=0$ on $K_{0}^{\prime}$, we see that

$$
\left\|\widetilde{p}^{x^{\phi(F)}}\right\|_{R_{2}}^{2} \leqq\left\|f \circ \phi^{-1}\right\|_{R_{2}}^{2} \leqq K(\phi)\|f\|_{R_{1}}^{2} \leqq K(\phi)\left\|\widetilde{p}^{x^{F^{*}}}\right\|_{R_{2}}^{2}
$$

Hence we obtain that $\widetilde{C}^{\prime}(\phi(E)) \leqq \widetilde{C}^{\prime}(\phi(F)) \leqq K(\phi) \widetilde{C}(F)$. Since $F$ is arbitrary, we obtain that $\widetilde{C}^{\prime}(\phi(E)) \leqq K(\phi) \widetilde{C}(E)$. On the other hand, since $\phi^{-1}$ is a quasiconformal mapping and $K\left(\phi^{-1}\right)=K(\phi)$, we see that $\widetilde{C}(E) \leqq K(\phi) \widetilde{C}^{\prime}(\phi(E))$. This completes the proof.

Corollary. For any Borel subset $A$ of $R_{1}-K_{0}$, we have

$$
1 / K(\phi) \widetilde{C}(A) \leqq \widetilde{C}^{\prime}(\phi(A)) \leqq K(\phi) \widetilde{C}(A)
$$

We denote by $d_{1}$ (resp. $d_{2}$ ) the metric defined in Definition 4 with respect to $R_{1}-K_{0}$ (resp. $R_{2}-K_{0}^{\prime}$ ). By the aid of Theorem 4 , we obtain the following theorem.

TheOREM 5. $1 / K(\phi) d_{1}(a, b) \leqq d_{2}(\phi(a), \phi(b)) \leqq K(\phi) d_{1}(a, b)$ for any $a, b \in$ $R_{1}-K_{0}$.

Corollary. Let $R$ be an open Riemann surface and let $K_{0}$ be a closed disk in $R$. If $\phi$ is a quasiconformal mapping of $R$ onto itself such that $\phi\left(K_{0}\right)=K_{0}$, then

$$
1 / K(\phi) d(a, b) \leqq d(\phi(a), \phi(b)) \leqq K(\phi) d(a, b)
$$

for any $a, b \in R-K_{0}$.

By a discussion similar to that in the proof of Theorem 4, we have the following.

Proposition 4. Let $R_{1}$ and $R_{2}$ be hyperbolic Riemann surfaces. If $\phi: R_{1} \rightarrow R_{2}$ is an onto quasiconformal mapping, then

$$
1 / K(\phi) \rho_{R_{1}}(a, b) \leqq \rho_{R_{2}}(\phi(a), \phi(b)) \leqq K(\phi) \rho_{R_{1}}(a, b)
$$

for any $a, b \in R_{1}$.

COROllary. Let $R$ be a hyperbolic Riemann surface. If $\phi$ is a quasiconformal mapping of $R$ onto itself, then

$$
1 / K(\phi) \rho_{R}(a, b) \leqq \rho_{R}(\phi(a), \phi(b)) \leqq K(\rho) \rho_{R}(a, b)
$$

for any $a, b \in R$.

Definition 5. Let $A$ be a non-empty subset of $R_{0}$. We define the diameter $\delta(A)$ of $A$ with respect to $d$ by $\sup _{a, b \in A} d(a, b)$. Furthermore we set $\delta(\emptyset)=0$.

3) See p. 185 in [2] for the definition of $\chi^{F}$. 
LEMMA 4. If $F$ is a connected closed snbset of $R_{0}$, then $\delta(F) \leqq \widetilde{C}(F)$.

\section{4. d-completions of open Riemann surfaces.}

Let $R$ be an open Rienann surface. Let $K_{0}$ be a cloded disk in $R$ and let $R_{0}=R-K_{0}$. We denote by $R_{N}^{*}$ (resp. $R_{D}^{*}$ ) the Kuramochi compactification (resp. the Royden compactification) of $R$. The Kuramochi boundary (resp. the Royden boundary) of $R$ is denoted by $\Delta_{N}$ (resp. $\Delta_{D}$ ) (cf. [2]). For a subset $A$ of $R$, we denote by $\bar{A}^{N}$ (resp. $\bar{A}^{D}$ ) the closure of $A$ in $R_{N}^{*}$ (resp. $R_{D}^{*}$ ). Let $R_{1}^{*}$ and $R_{2}^{*}$ be two compactifications of $R$. If there is a continuous mapping $\pi$ of $R_{1}^{*}$ onto $R_{2}^{*}$ whose restriction to $R$ is the identity and $\pi^{-1}(R)=R$, then $\pi$ is called a canonical mapping of $R_{1}^{*}$ onto $R_{2}^{*}$ and $R_{2}^{*}$ is called a quotient space of $R_{1}^{*}$. It is known (cf. [2]) that $R_{N}^{*}$ is a qüotient space of $R_{D}^{*}$.

We denote by $d_{B}$ the set of all Kuramochi boundary points $b$ such that the Kuramochi kernel $\tilde{g}_{b}$ with pole $b$ is bounded. The set $\Delta_{1} \cap \Delta_{B}$ is denoted by $\Delta_{\mathcal{S}}$ (cf. [2]). By definition, we see that $\Delta_{S} \subset \Delta_{B}$.

Definition 6. We denote by $U_{H M}$ the class of all open Riemann surfaces with $\Delta_{B} \neq \emptyset$ :

It is known that $O_{G} \cap U_{H M}=\emptyset$.

THEOREM 6. Suppose $R$ is an open Riemann surface with $R \notin U_{H M}$. If $D$ is an open disk in $R$ with $D \supset K_{0}$, then $R-D$ is totally bounded with respect to $d$.

5. Prö́F: (i) For $b \in \Delta_{1}$, let $F_{n}(b)=\left\{z \in R_{0} ; \tilde{g}_{b}(z) \geqq n\right\}(n=1,2, \cdots)$. First we shall prove that $\bigcup_{b \in A_{1}}{\overline{F_{n}}(b)^{D}}_{(n=1,2, \cdots)}$ is a neighborhood of $\Delta_{D}$ in $R_{D}^{*}$. Let $n$ be fixed. If this were not the case, then we could find $\xi \in \Delta_{D}$ such that $U(\xi)-\bigcup_{b \in A_{1}} \overline{F_{n}(b)} \neq \emptyset$ for any neighborhood $U(\xi)$ of $\xi$ in $R_{D}^{*}$. Let $\pi$ be the canonical mapping of $R_{D}^{*}$ onto $R_{N}^{*}$ and let $\pi(\xi)=b_{0}$. Let $\left\{V_{j}\left(b_{0}\right)\right\}_{j=1}^{\infty}$ be a sequence of open neighborhoods of $b_{0}$ in $R_{N}^{*}$ such that $V_{j}\left(b_{0}\right) \supset V_{j+1}\left(b_{0}\right)$

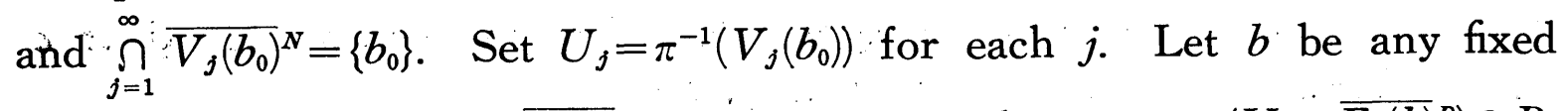
point of $\Delta_{1}$. Since $U_{j}-\overline{F_{n}(b)^{D}} \neq \emptyset$ for each $j$, there is $z_{j} \in\left(U_{j}-\bar{F}_{n}(b)^{D}\right) \cap R_{0}$ $\left(=V_{j}\left(b_{0}\right) \cap R_{0}-F_{n}(b)\right)$ for exach $j$. Then $\tilde{g}_{b}\left(z_{j}\right) \leqq n$ for each $j$. Since $a \rightarrow \tilde{g}_{b}(a)$ is lower semicontinuous on $R_{N}^{*}-K_{0}$, we obtain that $\tilde{g}_{b}\left(b_{0}\right) \leqq n$. Since $b$ is arbitrary, it follows from symmetry of the Kuramochi kernel and a domination principle (cf. Folgesatz 17.2 in [2]) that $\tilde{g}_{b_{0}} \leqq n$ on $R_{0}$. Thus $\Delta_{B} \neq \emptyset$ and this contradicts $R \notin U_{H M}$. Hence we see that $\bigcup_{b \in A_{1}}{\overline{F_{n}}}_{n}(b)^{D}$ is a neighborhood of $\Delta_{D}$ in $R_{D}^{*}$ for each $n$. 
(ii) For each $n$ and $b \in \Delta_{1}$, we can find an open subset $G_{n}(b)$ of $R_{D}^{*}$ with $\overline{F_{n+1}(b)^{D}} \subset G_{n}(b) \subset{\overline{F_{n}(b)}}^{D}$. By (i), we see that $\bigcup_{b \in A_{1}} G_{n}(b)$ is an open covering of $\Delta_{D}$. Hence we can find $b_{1}, \cdots, b_{k} \in \Delta_{1}$ such that $\bigcup_{j=1}^{k} G_{n}\left(b_{j}\right)$ is a neighborhood of $\Delta_{D}$ in $R_{D}^{*}$. It is easy to see that $\bigcup_{j=1}^{k} \overline{F_{n}\left(b_{j}\right)^{N}}$ is a neighborhood of $\Delta_{N}$ in $R_{N}^{*}$. Since $\delta\left(F_{n}\left(b_{j}\right)\right) \leqq \widetilde{C}\left(F_{n}\left(b_{j}\right)\right)=1 / n^{4)}$, we complete the proof.

COROllaRy. If $R$ is an open Riemann surface with $R \notin U_{H M}$, then the completion of $R-D$ with respect to $d$ is compact.

Let $R$ be an open Riemann surface and $K_{0}$ be a closed disk in $R$. Let $D$ be an open disk in $R$ with $D \supset K_{0}$. Since the completion of $R-D$ with respect to $d$ does not depend on the choice of $K_{0}$ (Lemma 1), we denote it by $(R-D)_{d}^{*}$. Furthermore we set $R_{d}^{*}=(R-D)_{d}^{*} \cup D$. If $R$ does not belong to $U_{H M}$, then it follows from the above corollary that $R_{d}^{*}$ is a compactification of $R$. We note that if $R=\{|z|<1\}$, then $R_{l}^{*}$ is homeomorphic to $\{|z| \leqq 1\}$.

THEOREM 7. If $R$ is an open Riemann surface with $R \notin U_{H M}$, then $R_{d}^{*}$ is a quotient space of $R_{D}^{*}$.

PRoOF. Let $E_{1}$ and $E_{2}$ be regular closed subsets of $R$ with $\bar{E}_{1}{ }^{D} \cap \bar{E}_{2}{ }^{D}$ $\neq \emptyset$. Let $\xi$ be a point of $\bar{E}_{1}{ }^{D} \cap \bar{E}_{2}{ }^{D}$. It follows from the proof of Theorem 6 that, for any $n$, there exists $a, b \in \Delta_{1}$ such that ${\overline{F_{n}}(b)}^{D}$ is a neighborhood of $\xi$ in $R_{D}^{*}$. Then $F_{n}(b) \cap E_{i} \neq \emptyset(i=1,2)$. Let $z_{i}$ be a point of $F_{n}(b) \cap E_{i}$ $(i=1,2)$. Then $0 \leqq d\left(z_{1}, z_{2}\right) \leqq \widetilde{C}\left(F_{n}(b)\right)=1 / n$. Since $n$ is arbitrary, we obtain that $\inf \left\{d\left(z_{1}, z_{2}\right) ; z_{1} \in E_{1}, z_{2} \in E_{2}\right\}=0$, that is, $\bar{E}_{1}^{d} \cap \bar{E}_{2}{ }^{d} \neq \emptyset\left(\bar{E}_{i}{ }^{d}\right.$ is the closure of $E_{i}$ in $R_{d}^{*}$ ). Thus it can be seen that $R_{d}^{*}$ is a quotient space of $R_{D}^{*}$.

THEOREM 8. If $R \in 0_{a}$, then $R_{d}^{*}$ is homeomorphic to the Kerékjárt'Stoilow's compactification $R_{K S}^{*}$ of $R$.

ProOF. First we note that $R_{d}^{*}$ is compact by the Corollary of Theorem 6. Let $e$ be any point of $\Delta_{K S}=R_{K S}^{*}-R$. Let $\left\{G_{n}\right\}_{n=1}^{\infty}$ be a determining sequence of $e$. Then each $G_{n}$ is a domain on $R$ with compact relative boundary $\partial G_{n}$ in $R$ and $G_{n+1} \cup \partial G_{n+1} \subset G_{n}(n=1,2, \cdots), \bigcap_{n=1}^{\infty} G_{n}=\emptyset$. Since $\delta\left(G_{n} \cup \partial G_{n}\right) \leqq \widetilde{C}\left(G_{n} \cup \partial G_{n}\right) \rightarrow 0$ as $n \rightarrow \infty, \bigcap_{n=1}^{\infty} \overline{G_{n} \cup \partial G_{n}}{ }^{a}$ is a single point in $R_{d}^{*}$, where ${\overline{G_{n} \cup \partial G_{n}}}^{d}$ is the closure of $G_{n} \cup \partial G_{n}$ in $R_{d}^{*}$. Thus we denote it by $\pi(e)$. For each $z \in R$ we set $\pi(z)=z$. Then we can show that $\pi$ is a continuous mapping of $R_{K S}^{*}$ onto $R_{d}^{*}$. Let $e_{1}$ and $e_{2}$ be any points of $\Delta_{K S}$ with

4) By the aid of Folgesatz 17. 22 in [2] we can prove the equality. 
$e_{1} \neq e_{2}$. Then we can find two domains $\Omega_{1}$ and $\Omega_{2}$ on $R$ such that

(1) $\partial \Omega_{1}$ and $\partial \Omega_{2}$ are compact in $R$,

(2) the closure $\bar{\Omega}_{i}{ }^{K S}$ of $\Omega_{i}$ in $R_{K S}^{*}$ is a neighborhood of $e_{i}$ in $R_{K S}^{*}$ $(i=1,2)$,

(3) $\bar{\Omega}_{1}{ }^{K S} \cap \bar{\Omega}_{2}{ }^{K S}=\emptyset$.

Since $0<d\left(\partial \Omega_{1}, \partial \Omega_{2}\right)=\inf \left\{d\left(z_{1}, z_{2}\right) ; z_{1} \in \partial \Omega_{1}, z_{2} \in \partial \Omega_{2}\right\} \leqq d(a, b)$ for any $a \in \Omega_{1}$ and $b \in \Omega_{2}$, we have $\pi\left(e_{1}\right) \neq \pi\left(e_{2}\right)$. Hence we see that $\pi$ is a homeomorphism of $R_{k S}^{*}$ onto $R_{i}^{*}$.

Combining Theorem 5 and Theorem 6 , we obtain the following theorem.

THEOREM 9. Let $R_{1}$ and $R_{2}$ be two open Riemann surfaces which do not belong to $U_{H M}$. If $\phi$ is a quasiconformal mapping of $R_{1}$ onto $R_{2}$, then $\phi$ can be homeomorphically extended to a mapping from $\left(R_{1}\right)_{i}^{*}$ onto $\left(R_{2}\right)_{i}^{*}$.

\section{References}

[1] N. Boboc and G. MocANU: Sur la notion de métrique harmonique sur une surface riemannienne hyperbolique, Bull. Math. de la Soc. Math. Phys., 5 (52), 1961.

[2] C. Constantinescu and A. CoRneA: Ideale Ränder Riemannscher Flächen, Springer Verlag, 1963.

[3] J. HERSCH: Longueurs extrémales et théorie des fonctions, Comment. Math. Helv., 29 (1955).

[4] L. SARIO and M. NAKAI: Classification theory of Riemann surfaces, Springer Verlag, 1970. 